\title{
100. On the Isomorphism of Topological Groups
}

\author{
By Takashi Kasuga \\ Department of Mathematics, Osaka University \\ (Comm. by K. Kunugi, M.J.A., Oct. 12, 1953)
}

In this note we shall prove some simple theorems on the identification of two topological groups with the same underlying abstract group.

1. Some notations and definitions. In the following we mean by the word "topology" a topology which satisfies Hausdorff's axioms.

We denote by $R(T)$ a set $R$ with topology $T$ (in this note $R$ may be an abstract set or an abstract group or an abstract linear space). For two sets $R_{1}$ and $R_{2}$ (without topologies) we denote by $R_{1} \times R_{2}$ their direct product, that is, the set of all pairs $\left(x_{1}, x_{2}\right)$ where $x_{1} \in R_{1}$ and $x_{2} \in R_{2}$. When $R_{1}$ and $R_{2}$ are both abstract groups or both abstract linear spaces, we can consider $R_{1} \times R_{2}$ as an abstract group or as an abstract linear space in the well-known manner (in the case of groups, we define $\left(x_{1}, x_{2}\right)\left(y_{1}, y_{2}\right)$ by $\left(x_{1} y_{1}, x_{2} y_{2}\right)$ where $x_{1}, y_{1} \in R_{1}$ and $x_{2}, y_{2} \in R_{2}$, and in the case of linear spaces, we define $\left(x_{1}, x_{2}\right)+\left(y_{1}, y_{2}\right)$ and $\alpha\left(x_{1}, x_{2}\right)$ by $\left(x_{1}+y_{1}, x_{2}+y_{2}\right)$ and $\left(\alpha x_{1}, \alpha x_{2}\right)$ respectively where $x_{1}, y_{1} \in R_{1} x_{2}, y_{2} \in R_{2}$ and $\alpha$ is any real number). When $R_{1}\left(T_{1}\right)$ and $R_{2}\left(T_{2}\right)$ are two topological spaces, we denote by $R_{1}\left(T_{1}\right) \times R_{2}\left(T_{2}\right)$ the so-called topological direct product of $R_{1}\left(T_{1}\right)$ and $R_{2}\left(T_{2}\right)$. We denote the topology of the topological space $R_{1}\left(T_{1}\right) \times$ $R_{2}\left(T_{2}\right)$ by $T_{1} \times T_{2}$. Evidently by the definitions $R_{1} \times R_{2}\left(T_{1} \times T_{2}\right)=$ $R_{1}\left(T_{1}\right) \times R_{2}\left(T_{2}\right)$. For a subset $S$ of a topological space $R(T)$, we denote by $S\{T\} S$ with the topology induced by $T$. If $R$ is endowed with two topologies $T$ and $T^{*}$, and $T$ is stronger (that is, with more open sets) than $T^{*}$ or at least equivalent to $T^{*}$, then we write $T \geqq T^{*}$. By $A_{R}$, we denote the diagonal of $R \times R$, that is, the set of the elements of $R \times R$ which are of the form $(a, a)$ where $a \in R$. When $R$ is an abstract group or an abstract linear space, $J_{R}$ is a subgroup or a linear subspace of $R \times R$ respectively.

In the following, we shall say that a topological space is semicompact, if it is locally bicompact and can be represented as a sum of a number, countable at most, of bicompact sets.

2. We prove first a simple lemma.

Lemma 1. If $R$ is endowed with three topologies $T_{1}, T_{2}, T^{*}$ and $T_{1} \geqq T^{*}, T_{2} \geqq T^{*}$, then $J_{R}$ is closed in $R\left(T_{1}\right) \times R\left(T_{2}\right)$.

Proof. $T_{1} \times T_{2} \geqq T^{*} \times T^{*}$, since $T_{1} \geqq T^{*}$ and $T_{2} \geqq T^{*}$. On the other hand, $\Delta_{R}$ is closed in $R \times R\left(T^{*} \times T^{*}\right) \quad\left(=R\left(T^{*}\right) \times R\left(T^{*}\right)\right)$, as 
$R\left(T^{*}\right)$ is a Hausdorff space. Hence $A_{R}$ is closed in $R \times R\left(T_{1} \times T_{2}\right)$ $\left(=R\left(T_{1}\right) \times R\left(T_{2}\right)\right)$.

3. Theorem 1. Let $G\left(T_{1}\right)$ and $G\left(T_{2}\right)$ be both semi-compact topological groups with the same underlying abstract group $G$. If we can define a topology $T^{*}$ on $G$ such that $T_{1} \geqq T^{*}$ and $T_{2} \geqq T^{*}\left(G\left(T^{*}\right)\right.$ needs not be necessarily a topological group), then $G\left(T_{1}\right)$ and $G\left(T_{2}\right)$ are one and the same topological group.

Proof. From the assumption that $G\left(T_{1}\right)$ and $G\left(T_{2}\right)$ are both semi-compact topological groups, we can easily prove that $G\left(T_{1}\right) \times$ $G\left(T_{2}\right)$ is also a semi-compact topological group. On the other hand, by Lemma $1, \Delta_{G}$ is closed in $G\left(T_{1}\right) \times G\left(T_{2}\right)$ as $T_{1} \geqq T^{*}$ and $T_{2} \geqq T^{*}$. Hence $\Delta_{G}\left\{T_{1} \times T_{2}\right\}$ (that is, $\Delta_{G}$ with the topology induced by the topology $T_{1} \times T_{2}$ of $\left.G\left(T_{1}\right) \times G\left(T_{2}\right)\right)$ is a semi-compact topological group.

The mapping $(a, a) \rightarrow a$, where $a \in G$, is continuous as a mapping of $\Delta_{G}\left\{T_{1} \times T_{2}\right\}$ onto $G\left(T_{1}\right)$ as well as, as a mapping of $J_{G}\left\{T_{1} \times T_{2}\right\}$ onto $G\left(T_{2}\right)$ and at the same time it is an abstract group isomorphism of $\Delta_{G}$ onto $G$. It is well-known that if an abstract group homomorphism of a semi-compact topological group onto another semi-compact topological group is continuous, then this mapping is open $^{1)}$. Since $G\left(T_{1}\right), G\left(T_{2}\right)$ and $\Delta_{G}\left\{T_{1} \times T_{2}\right\}$ are semi-compact topological groups, the mapping $(a, a) \rightarrow a$ where $a \in G$, is an isomorphism of $\Delta_{G}\left\{T_{1} \times T_{2}\right\}$ onto $G\left(T_{1}\right)$ and is at the same time an isomorphism of $\Delta_{G}\left\{T_{1} \times T_{2}\right\}$ onto $G\left(T_{2}\right)$. Hence the identity mapping $a \rightarrow a$ where $a \in G$, is an isomorphism of $G\left(T_{1}\right)$ onto $G\left(T_{2}\right)$. Thus Theorem 1 is proved.

4. From Theorem 1 we can easily deduce the two following theorems.

Theorem 2. Let $R\left(T^{\prime}\right)$ be a topological space and $G$ be a group of transformations of $R$ as an abstract set (we denote by $g(p)$ the result of the transformation $g(\epsilon G)$ as applied to $p(\in R)$ ). If we can define a topology $T$ on $G$ such that $G(T)$ is a semi-compact topological group and the mapping $A_{p} g \rightarrow g(p)$ where $g \in G$ and $p$ is a fixed element of $R$, is continuous as a mapping of $G(T)$ into $R\left(T^{\prime}\right)$ for any fixed element $p$ of $R$, then such $T$ is uniquely determined.

Proof. We can easily construct the weakest topology $T^{*}$ among the topologies $T^{* *}$ of $G$ such that the mapping $A_{p} g \rightarrow g(p)$ is continuous as a mapping of $G^{\prime}\left(T^{* *}\right)$ into $R\left(T^{\prime}\right)$ for any fixed element $p$ of $R\left(G\left(T^{* *}\right)\right.$ needs not be necessarily a topological group).

When $p_{1}, \ldots, p_{n}$ are elements of $R$ and $g_{0}$ is an element of $G$ and $U_{1}, \ldots, U_{n}$ are neighbourhoods in $R\left(T^{\prime}\right)$ of $g_{0}\left(p_{1}\right), \ldots, g_{0}\left(p_{n}\right)$ respectively, we denote by $V\left(g_{0}: p_{1}, \ldots, p_{n}: U_{1}, \ldots, U_{n}\right)$ the set of the elements $g$ of $G$ such that $g\left(p_{i}\right) \in U_{i} i=1, \ldots, n$. 
We can define the topology $T^{*}$ of $G$ by taking $V\left(g_{0}: p_{1}, \ldots, p_{n_{2}}\right.$ : $U_{1}, \ldots, U_{n}$ ) for any finite number of elements $p_{1}, \ldots, p_{n}$ of $R$ and for any neighbourhoods $U_{1}, \ldots, U_{n}$ in $R\left(T^{\prime}\right)$ of $g_{0}\left(p_{1}\right), \ldots, g_{0}\left(p_{n}\right)$ respectively as the neighbourhoods of any element $g_{0}$ of $G$. Hausdorff's axioms can be easily verified for this system of neighbourhoods. Then for any $T$ which satisfies the conditions of Theorem $2, T \geq T^{*}$. Hence by Theorem 1, $T$ is uniquely determined.

Theorem 3. Let $H$ be a subgroup of an abstract group $G$ and $G\left(T^{\prime}\right)$ be a topological group with the underlying abstract group $G$. If we can define on $H$ a topology $T$ such that $H(T)$ is a semi-compact topological group and the identity mapping: $h \rightarrow h$ where $h \in H$, is continuous as a mapping of $H(T)$ into $G\left(T^{\prime}\right)$, then $T$ is uniquely determined.

Proof. If we denote by $T^{*}$ the topology induced on $H$ by the topology $T^{\prime}$ of $G\left(T^{\prime}\right)$, then for any $T$ which satisfies the conditions of Theorem $3, T \geqq T^{*}$. Hence by Theorem $1, T$ is uniquely determined.

5. Theorem 4. Let $L\left(N_{1}\right)$ and $L\left(N_{2}\right)$ be two Banach spaces with the norms $N_{1}$ and $N_{2}$ respectively, but with the same underlying abstract linear space $L$. If the weak topologies of $L\left(N_{1}\right)$ and $L\left(N_{2}\right)$ are equivalent, then $L\left(N_{1}\right)$ and $L\left(N_{2}\right)$ are isomorphic by the identity mapping. (Roughly speaking, a Banach space is determined in its topological structure by its algebraic structure and its weak topology.)

Proof. We denote by $T_{1}$ and $T_{2}$ the topologies on $L$ induced by the norms $N_{1}$ and $N_{2}$ respectively and by $W$ the common weak topology of $L\left(N_{1}\right)$ and $L\left(N_{2}\right)$. By defining a suitable norm (not uniquely determined) on $L \times L$, we can consider the linear space $L \times L$ as a Banach space whose topology is $T_{1} \times T_{2}$. We denote this norm by $N_{1} \times N_{2}$ and this Banach space by $L \times L\left(N_{1} \times N_{2}\right)$ or by $L\left(N_{1}\right) \times L\left(N_{2}\right)$.

By Lemma 1, $J_{L}$ is a closed linear subspace of $L\left(N_{1}\right) \times L\left(N_{2}\right)$ as $T_{1} \geqq W$ and $T_{2} \geqq W$. Hence we can consider $\Delta_{L}$ as a Banach space with the norm induced on $d_{L}$ by the norm $N_{1} \times N_{2}$ of $L\left(N_{1}\right) \times$ $L\left(N_{2}\right)$. We denote this Banach space by $\Delta_{L}\left\{N_{1} \times N_{2}\right\}$.

The mapping $(a, a) \rightarrow a$ where $a \in L$, is continuous as a mapping of $d_{L}\left\{N_{1} \times N_{2}\right\}$ onto $L\left(N_{1}\right)$, as well as, as a mapping of $d_{L}\left\{N_{1} \times\right.$ $\left.N_{2}\right\}$ onto $L\left(N_{2}\right)$ and at the same time, it is an abstract linear isomorphism of $\Delta_{L}$ onto $L$. Moreover $\Delta_{L}\left\{N_{1} \times N_{2}\right\}, L\left(N_{1}\right)$ and $L\left(N_{2}\right)$ are all Banach spaces. Hence by a well-known theorem of Banach ${ }^{2)}$, the mapping $(a, a) \rightarrow a$ where $a \in L$, is an isomorphism of $J_{L}\left\{N_{1} \times\right.$ $\left.N_{2}\right\}$ onto $L\left(N_{1}\right)$ and is at the same time an isomorphism of $J_{L}\left\{N_{1} \times\right.$ $N_{2}$ \} onto $L\left(N_{2}\right)$. Then the mapping $a \rightarrow a$ where $a \in L$, is an isomorphism of $L\left(N_{1}\right)$ onto $L\left(N_{2}\right)$. Thus Theorem 4 is proved. 


\section{Notes}

1) Cf. T. Tannaka, Isogunron (in Jap.), 36-37 (1949). Also cf. H. Freudenthal, Einige Sătze über topologische Gruppen, Ann. Math., 37 (1936), where this proposition is proved, under the assumption that both topological groups are separable.

2) Cf. Banach, Théorie des opérations linéaires, 41 (1932). 